ORIGINAL

\title{
Resultados y reflexiones del Comité Científico del XXVIII Congreso de la Sociedad Española de Calidad Asistencial
}

\author{
M. Santiñá* y D. Martín-Rodriguez \\ Comité Científico, Hospital Clínic, Barcelona, España
}

\section{PALABRAS CLAVE \\ Evaluación; \\ Calidad Asistencial; \\ Congreso}

\section{KEYWORDS}

Assessment;

Quality in

Healthcare;

Conference

\begin{abstract}
Resumen
Objetivos: Valorar el cumplimiento de los objetivos encomendados al comité científico (CC) y conocer si los resultados obtenidos han variado respecto a anteriores congresos de la Sociedad Española de Calidad Asistencial (SECA).

Métodos: Descripción cronológica del trabajo del CC y comparación con los resultados de los dos últimos congresos de la SECA.

Resultados: Ochocientos sesenta y nueve trabajos presentados, de los cuales el 32,6\% correspondían al área temática de seguridad de pacientes. Galicia fue la comunidad que más comunicaciones presentó $(28,0 \%)$, seguida de Cataluña $(12,9 \%)$ y Murcia $(10,9 \%)$.

Conclusiones: La metodología seguida por el CC facilitó el cumplimiento de los plazos previstos en el manual de congresos de la SECA y ayudó a alcanzar los objetivos del mismo, estructurando las presentaciones de los trabajos según su nivel de calidad. Se constata un año más las características de congreso multidisciplinar, abierto a los países del área latinoamericana, por el número de comunicaciones presentadas y su distribución por estamentos, comunidades autónomas y países que las presentan.

(c) 2011 SECA. Publicado por Elsevier España, S.L. Todos los derechos reservados.
\end{abstract}

Results and reflections of the Scientific Committee of the XXVIII Conference of the Spanish Society of Quality in Healthcare

\begin{abstract}
Objectives: To determine whether the objectives entrusted to the Scientific Committee (SC) of the 28th National Conference have been met, and to determine whether the results differed from those of previous conferences.

Methods: A chronological description of the work of the SC and a comparison with the results of the previous two Conferences of the Spanish Society for Quality in health care (SSQHC).
\end{abstract}

\footnotetext{
* Autor para correspondencia. Correo electrónico: msantina@clinic.ub.es (M. Santiñá).
} 
Results: A total of 869 papers were submitted, of which $32.6 \%$ corresponded to the area of patient safety. Galicia was the community that presented more communications $(28 \%)$, followed by Catalonia $(12.9 \%)$ and Murcia $(10.9 \%)$.

Conclusions: The methodology used by the SC helped in the compliance with the deadlines in the SSQHC Conference manual and contributed to achieve its objectives. Abstract presentations were classified accordingly to their quality. The Conference has been consolidated as a multidisciplinary meeting with participation by doctors, nurses, managers and open to the Latin-American community.

La Sociedad Española de Calidad Asistencial (SECA) se constituyó como asociación profesional científica multidisciplinar en el año 1982, con el objetivo de promocionar la mejora de la calidad en los centros sanitarios. Desde su creación organiza una reunión anual en forma de congreso para presentar y debatir los temas más relevantes en este campo del conocimiento. En el año 2010 tuvo lugar en Santiago de Compostela su vigesimoctava edición, teniendo como anfitriona a la Sociedad Gallega de Calidad Asistencial (SGQA).

Una vez finalizado el congreso de la SECA es habitual realizar un balance del mismo ${ }^{1--8}$, por lo que los objetivos del presente trabajo son: 1 . Conocer si se han cumplido los objetivos encomendados al comité científico (CC) y 2 . Conocer si los resultados obtenidos han variado respecto a los anteriores congresos de la SECA.

\section{Material y método}

La preparación de un congreso tuvo dos vertientes, la organizativa y la científica. La científica se desarrolló en 3 etapas: precongresual, congresual y poscongresual.

En la etapa precongresual se preparó la estructura del programa científico y sus contenidos, para ello, la SECA nombró un comité (CC), con un/a presidente/a al frente que cuenta con el apoyo de un/a secretario/a, un vocal de enlace entre comités y una serie de vocales, hasta un máximo de 24.

Se establecieron unos objetivos generales, que el caso del 28 Congreso fueron mejorar la calidad científica del congreso de la SECA y colaborar con el comité organizador (CO) para conseguir el adecuado equilibrio económico. A partir de ello se fijaron unos objetivos de trabajo con un calendario, de acuerdo con lo que prevé el manual de congresos de la SECA 9 para esta etapa precongresual.

El presidente responsable de conseguir esos objetivos actuó de dinamizador del CC.

La primera reunión del CC se realizó en Sevilla, aprovechando las fechas de celebración del vigesimoséptimo congreso en el mes de octubre. Esta reunión permitió que los componentes del CC se conociesen personalmente, realizar una primera sesión de ideas sobre las que trabajar y poner en común los objetivos a alcanzar y el método de trabajo del grupo. En este caso y dada la dispersión geográfica de los miembros del CC, se optó por el correo electrónico como medio de transmisión de información y de debate de los diferentes temas que el presidente iría proponiendo al CC.

A partir de esta reunión, semanalmente el presidente anunciaba el tema a tratar en esa semana, introduciendo una serie de preguntas que el resto de miembros del CC tenía que responder con una fecha límite para hacerlo. Recogía las respuestas y reflexiones hasta alcanzar el máximo consenso en cada uno de los temas expuestos.

En todo momento se siguió el cronograma de actividades previsto, aunque la evaluación de trabajos sufrió un recorte en su tiempo pues el CO alargó el período de aceptación de resúmenes dadas las peticiones que había recibido en este sentido.

En octubre se realizó el congreso (etapa congresual) y durante el mismo se solicitó a los congresistas que al finalizar entregaran cumplimentada la encuesta de opinión a fin de conocer sus impresiones en cuanto a organización y desarrollo del programa científico.

La etapa poscongresual sirvió para elaborar una memoria de cierre del congreso.

\section{Resultados}

Se consiguió cumplir el cronograma de actividades previsto, excepto la fecha límite para la recepción de comunicaciones ${ }^{10}$ que se alargó dos semanas.

Se enviaron 932 trabajos para su presentación en el mismo. Los que obtuvieron una puntuación por encima de 3,5 puntos se aceptaron, en total $869(93,2 \%)$, y se rechazaron $63(6,8 \%)$.

Por áreas temáticas $32,6 \%$ trabajos sobre seguridad de los pacientes; $20,8 \%$ sobre procesos asistenciales; $12,6 \%$ sobre ciudadano $12,0 \%$ sobre sistemas de gestión; $11,0 \%$ sobre investigación/formación en calidad; $8,0 \%$ sobre liderazgo y $2,3 \%$ sobre responsabilidad social corporativa ${ }^{11}$.

Los trabajos con aquellas puntuaciones más altas $(\geq 8,2)$ se seleccionaron para cada una de las tres mesas de mejores experiencias, en total 12 trabajos; $252(27,2 \%)$ se presentaron en las mesas de comunicaciones orales; 205 (22,0\%) se defendieron en las mesas de defensa oral de póster y 393 $(42,5 \%)$ se presentaron en formato de póster electrónico.

Al congreso acudieron 924 congresistas provenientes de las diferentes comunidades autónomas (CC. AA.) de España y también de algunos países latinoamericanos.

Se solicitó por primera vez acreditación docente al sistema acreditador de formación continuada de las 


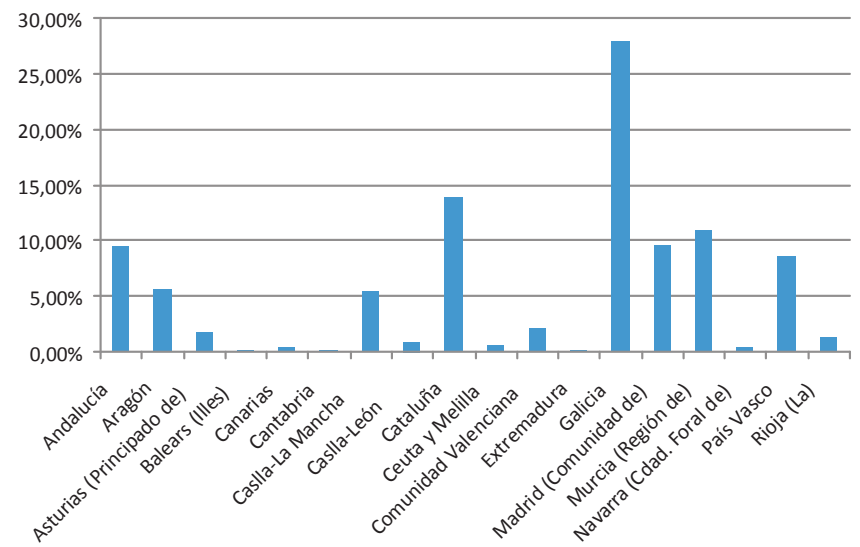

Figura 1 Porcentaje de trabajos presentados por comunidad autónoma en el 28 Congreso (porcentaje sobre el total de trabajos).

profesiones sanitarias para los 7 talleres que se realizaron, en los cuáles participaron un total de 153 alumnos. Mientras que a las actividades de desayunos con expertos acudieron 70 congresistas.

Por CC. AA., Galicia con 261 trabajos (28,0\%), Cataluña con $120(12,9 \%)$ y Murcia con $102(10,9 \%)$ fueron las que más comunicaron (fig. 1).

Los profesionales de la medicina con 260 trabajos $(27,9 \%)$ y de la enfermería con 250 (26,8\%) fueron los que enviaron más comunicaciones, aunque hubo profesionales de otras disciplinas sanitarias con $49(5,3 \%)$ y no sanitarias con 86 $(9,2 \%)$ que también participaron activamente.

Los profesionales del medio hospitalario fueron los que presentaron más comunicaciones, $402(43,1 \%)$, seguidos por los que trabajaban en la asistencia primaria, con 111 $(11,9 \%)$. El resto se repartió en otros ámbitos de actividad, como servicios centrales de la administración con 54 (5,8\%), salud mental con $12(1,3 \%)$ o servicios de emergencia con 19 $(2,0 \%)$.

Se recogieron un total de 254 encuestas en las que los congresistas manifestaron su satisfacción con el contenido científico del congreso (3,6 puntos en una escala de 5) y con su organización $(4,1 / 5)$ y donde expusieron lo que más y lo que menos les había gustado. En el primer punto destacaba la organización, la payasa que actuó, la variedad de los temas y los ponentes que intervinieron. En el segundo el número excesivo de comunicaciones, las entradas y salidas de las salas de comunicaciones, la selección de comunicaciones y el cumplimiento de los horarios.

\section{Discusión}

La metodología seguida por el CC facilitó el cumplimiento de los plazos previstos en el manual de congresos de la SECA ${ }^{9}$ para el buen desarrollo del congreso por lo que es recomendable que en próximos congresos se tenga en cuenta.

El 28 Congreso cumplió con los objetivos que se habían fijado, se consiguió una buena participación de congresistas provenientes de todas las CC. AA. Comparando el número de comunicaciones presentadas por cada una de ellas, en relación a los dos congresos anteriores (fig. 2) se observa

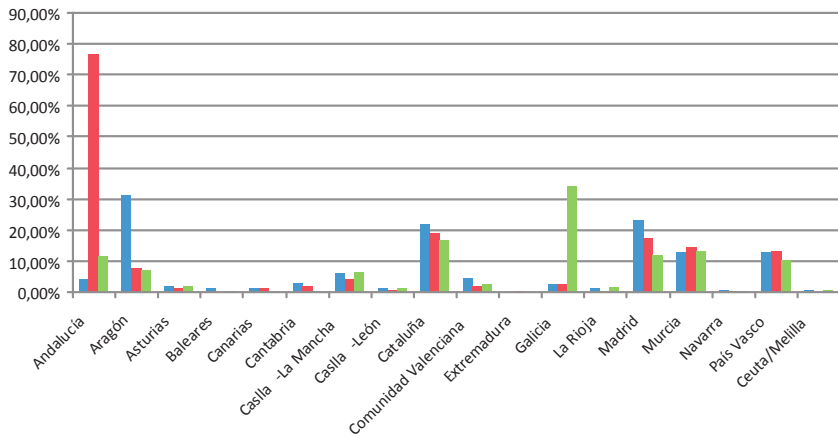

Figura 2 Evolución de los trabajos presentados por cada comunidad autónoma en los 3 últimos congresos de la SECA (porcentaje sobre el total de trabajos presentados).

Columna en azul: Congreso 2008 (Zaragoza)

Columna en rojo: Congreso 2009 (Sevilla)

Columna en verde: Congreso 2010 (Santiago de Compostela).

que se mantiene la tradición que aquella comunidad que organiza el congreso es la que más comunicaciones presenta, siendo Cataluña, Murcia, Madrid y País Vasco las que más trabajos comunican habitualmente $e^{5,7}$.

Una de las peticiones de los congresistas, recogidas en anteriores congresos ${ }^{5---8}$, era mejorar el nivel científico del mismo. Para tratar de conseguirlo, se orientó el esfuerzo en la mejor selección de comunicaciones, estructurando las sesiones en diferentes niveles de manera que las comunicaciones excelentes, de acuerdo con las puntuaciones otorgadas por los evaluadores del CC, tuviesen una mayor relevancia, incluyéndolas en sesiones plenarias (mejores experiencias); las más notables fuesen presentadas en las sesiones de comunicaciones orales o en sesiones de presentación de póster con defensa oral y el resto de las aceptadas en los paneles electrónicos de póster, teniendo en cuenta además que no se aceptó el $6,8 \%$ de los trabajos presentados, menos que en Zaragoza $(9,1 \%)$ con el mismo punto de corte y más que en de Sevilla $(1,5 \%)$.

Las dificultades que encuentra el CC del congreso para mejorar su nivel científico, algo que constantemente se solicita en las encuestas de opinión, es que la evaluación y por ende la aceptación o no del trabajo, se realiza sobre la base del resumen que envían los autores y aquí la diversidad de estilos y de habilidades es variada. Se pueden encontrar autores con una gran capacidad redactora y otros que no tanto, lo cual puede o no estar relacionado con la calidad del trabajo. Así mismo puede suceder que a la capacidad redactora del profesional se aúne la capacidad relatora, pero puede que no sea así, con lo cual habrá excelentes trabajos que serán bien defendidos y otros que no, con la consiguiente variación en la percepción de los congresista. Todo ello es lo que sucede en un congreso como el de SECA en que uno de sus rasgos distintivos es la de ser un congreso multidisciplinar, con diferentes niveles de conocimientos, experiencias y habilidades, pero con un compromiso común en la mejora de la calidad asistencial de las organizaciones sanitarias.

La variabilidad en el proceso por parte de los miembros del CC no es desdeñable, para evitarla en la medida de lo posible se realizó un cálculo de la desviación de cada puntuación efectuada por un evaluador respecto a su media de 
puntuaciones. Con este dato y la del otro evaluador se calculaba la diferencia que había en cada uno de los trabajos, si esta era superior a 3, se pedía a un tercer evaluador que lo puntuase. Con ello se trató de minimizar esa variabilidad que como en congresos anteriores ${ }^{5}$ es uno de los aspectos en que hay que seguir trabajando para disminuir ese $20 \%$ de casos en que se da.

Por otro lado el CC cada año, y este no fue una excepción, se encuentra con que a priori dispone de un mes para evaluar los aproximadamente mil trabajos que se presentan al congreso, pero la realidad es que estos treinta días se pueden llegar a reducir a la mitad pues es tradicional que el plazo de presentación de trabajos se alargue. Si no se hace así, los potenciales congresistas reclaman, pues todo el mundo lo espera, la mayoría hasta el plazo ampliado para enviar trabajos. Esta reducción del tiempo de evaluación dificulta el trabajo del CC pues al disponer de menos tiempo sufre de una mayor presión para poder cumplir con los plazos que le exige el $\mathrm{CO}$, pues hay que comunicar la aceptación de comunicaciones, hay que decidir en qué sesión se incluye cada comunicación, comunicárselo a los autores y cerrar el libro de ponencias.

Otro aspecto a tener en cuenta es que la organización debe favorecer la presentación de trabajos, pues se trata de un congreso abierto a la participación de profesionales de muy diversa procedencia, con diferentes perfiles y experiencia profesional, es por ello que se acaban aceptando trabajos con una puntuación por debajo de los 5 puntos ${ }^{7,8}$.

En esta edición se mantuvo el volumen de trabajos presentados ${ }^{11}$ y el nivel de presentación por CC. AA., todas presentaron trabajos e incluso se recibieron trabajos de países del área latinoamericana, con lo que el deseo de ser un congreso referente para los profesionales que trabajan en la mejora de la calidad asistencial en esos países, se debiera ir desarrollando y consolidando.

En el desarrollo de este congreso se ha tratado no solo de cumplir con los objetivos fijados por la Junta directiva de SECA y de seguir el manual de Congresos de SECA. También se procuró mejorar alguno de los aspectos que se habían planteado en ediciones anteriores ${ }^{7,8}$, como dar a conocer a los miembros del CC de una manera explícita su cometido concreto en el proceso de gestión científica del congreso, en Zaragoza ${ }^{7}$ se propuso realizar una aceptación formal de pertenencia en la que se concretaran unos compromisos mínimos con el presidente del comité. En Santiago no se ha seguido el modelo propuesto, pero sí se solicitó formalmente la aceptación de su participación en el CC. Hubo algún candidato que honestamente lo desestimó por considerar que no podía responder a lo que se le iba a exigir por tener otro compromiso previo. Se mantuvo una comunicación semanal intensa con los diferentes miembros del CC y con ello se consiguió una buena participación e implicación en la definición de los contenidos de su trabajo, en el proceso de evaluación de comunicaciones y en la moderación de sesiones.

Uno de los puntos identificados ${ }^{7,8}$ como área de mejora fue la revisión de los criterios de evaluación de las comunicaciones en aras de la simplificación y la efectividad para conseguir un buen nivel de calidad científica. En este caso los criterios han sido sencillos ${ }^{10}$, siguiendo la estructura lógica de los diferentes apartados de la comunicación científica.
Otro aspecto que cada año suscita debate y opiniones encontradas es el del número de comunicaciones que se presentan simultáneamente. Este año se redujo respecto al congreso anterior a un máximo de 7 salas de comunicaciones orales simultáneas y 3 de comunicaciones póster con defensa oral. Reduciéndose las presentaciones orales de póster y ajustando el número de comunicaciones orales, tal y como se recomendaba en las conclusiones del congreso de Sevilla.

También la recomendación de reducir las presentaciones encadenadas sobre diferentes partes de un mismo proyecto, por ello se redujo a 2 el número de comunicaciones que se podían enviar al congreso por autor y se advirtió que cuando se detectaran «trabajos encadenados» ${ }^{12}$ se informaría al autor que debería englobarlas todas en una única comunicación. Este aspecto incidió también en otra área de mejora detectada, la posible simultaneidad de un mismo autor presentando varias comunicaciones.

El 27,5\% de los congresistas respondió a la encuesta de opinión, lo cual puede inducir un sesgo en el análisis de las respuestas, pues no deja de ser un bajo porcentaje, aunque sea similar a los recogidos en otros congresos. La valoración del contenido científico y de la organización del congreso fue semejante a la obtenida en Zaragoza ${ }^{7}$.

Por todo ello consideramos que el 28 Congreso de Santiago de Compostela ha mantenido el nivel científico de los anteriores congresos, consolidando la presentación de pósters en formato electrónico y limitando la defensa oral de los trabajos a los más destacados, de entre los presentados, de cada modalidad.

\section{Conflicto de intereses}

Manuel Santiñá y Dolores Martín han elaborado conjuntamente este artículo que no ha tenido ningún tipo de financiación.

\section{Agradecimientos}

A todos los miembros del Comité Científico y Comité Organizador que trabajaron en la preparación y desarrollo del 28 Congreso, muchas gracias por su esfuerzo y buen hacer.

\section{Bibliografía}

1. Nualart L, Carbonell JM, Guix J, Serra J, Delgado R. El XV Congreso de la SECA en cifras: unos datos para la reflexión. Rev Calid Asist. 1997;12:419---24.

2. Sánchez E, Mozola J, Ansorena L, Lorenzo S. XVI Congreso de la SECA. Santander, 13-16 de octubre de 1998. Rev Calid Asist. 1998;13:468---72.

3. Parra P, Hernando L, Gomis R, Saturno PJ, Más A, Ramón T, et al. El Congreso de Murcia: análisis de resultados. Rev Calid Asist. 2002;17:43---9.

4. Lorenzo S, Cañibano S, Carbonell JM, Mira JJ, Peiró S, Fernández-León, et al. La gestión del Comité Científico del XXI Congreso de la Sociedad Española de Calidad Asistencial. Rev Calid Asist. 2004;19:27---33.

5. Parra P, López-Picazo JJ, Ramón T, Gomis R. Evolución de la producción científica en calidad. Análisis y mejora del proceso de evaluación en el XXIV Congreso de la Sociedad 
Española de Calidad Asistencial. Rev Calid Asist. 2008;23: 65---71.

6. Astier Peña MP, Lorenzo Martínez S, Santiñá M, Martín A. Desarrollo y aportaciones del Comité Científico del XXV Congreso de la Sociedad Española de Calidad Asistencial en Barcelona, 2007. Rev Calid Asist. 2009;24:207---14.

7. Astier Peña MP, Barrasa-Villar I, Garcia-Mata JR, Aranaz-Andrés J, Enriquez-Martín N, Vela-Marquina ML. XXVI Congreso de la Sociedad Española de Calidad Asistencial: un buen equilibrio entre calidad e innovación, ciencia y participación. Rev Calid Asist. 2010;25:291---300.

8. Reyes-Alcaraz V, Torres-Olivera A, Suárez-Alemán G. Evaluación y aprendizaje del 27 Congreso de la Sociedad Española de Calidad Asistencial. Rev Calid Asist. 2010;25:301---7.
9. Sociedad Española de Calidad Asistencial. Manual de Congresos de la Sociedad Española de Calidad Asistencial. 2006.

10. XXVIII Congreso de la Sociedad Española de Calidad Asistencial. Normas de Envío de comunicaciones y Normas de evaluación. [Consultado 16 May 2011]. Disponible en: http://congresocalidadasistencial.com/comunicacionesnormas.asp.

11. Libro de Ponencias y Comunicaciones del XXVIII Congreso de la SECA. Edita Sociedad Española de Calidad Asistencial, 2010. [Consultado 16 May 2011]. Disponible en: http://www. calidadasistencial.es/index. php?page=congresos ant.

12. Norman I, Griffiths P. Duplicate publication and 'salami slicing': ethical issues and practical solutions. Int J Nurs Stud. 2008;45:1257---60. 